Research Article

\title{
Study on the Relationships between Eigenmodes, Natural Modes, and Characteristic Modes of Perfectly Electric Conducting Bodies
}

\author{
Shaode Huang $\mathbb{D}$, Jin Pan, and Yuyue Luo \\ School of Electronic Science and Engineering, University of Electronic Science and Technology of China, Chengdu 611731, China \\ Correspondence should be addressed to Shaode Huang; shaodehuang@std.uestc.edu.cn
}

Received 30 October 2017; Revised 15 January 2018; Accepted 20 February 2018; Published 10 April 2018

Academic Editor: Miguel Ferrando Bataller

Copyright (C) 2018 Shaode Huang et al. This is an open access article distributed under the Creative Commons Attribution License, which permits unrestricted use, distribution, and reproduction in any medium, provided the original work is properly cited.

\begin{abstract}
We specify the corresponding relationships between eigenmodes, natural modes, and characteristic modes of perfectly electric conducting (PEC) bodies. The internal resonant frequencies, external resonant frequencies, current distributions, and radiation patterns of the three kinds of modes are compared to identify the relationships. Firstly, we review the definitions of the three kinds of modes and discuss their characteristics of the electromagnetic power. After that, we illustrate the relationships between these modes with three typical structures, that is, the infinite circular cylinder, sphere, and rectangular plate.
\end{abstract}

\section{Introduction}

The fields radiated or scattered by perfectly electric conducting (PEC) bodies can be analyzed using modal analysis methods. When the surfaces of PEC bodies coincide with coordinate surfaces, the modes have closed forms [1]. Otherwise, only numerical results can be obtained through numerical methods such as the method of moments (MoM) [2]. Eigenmode expansion method (EEM) [3], singularity expansion method (SEM) [4], and characteristic mode analysis (CMA) [5] are three common modal analysis methods in electromagnetic engineering. The three modal analysis methods result in three different kinds of modes generally, that is, eigenmodes, natural modes, and characteristic modes, respectively.

The EEM expands the currents and fields by the eigenfunctions of the integral equation kernels [3]. The currents and fields which relate to the eigenfunctions are called eigenmodes. The eigenmodes diagonalize the integral equation kernels and are independent of incident field [6].

The SEM arose from the observation that the transient response of electromagnetic scatterers appeared to be dominated by several damped sinusoids [4]. In complex frequency plane, these damped sinusoids are corresponding to the poles of the Laplace-transformed response. The SEM characterizes the object response (time domain) regarding all the singularities in the complex frequency plane [4]. These singularities in the complex frequency plane are called natural resonant frequencies, which are generally complex due to radiative loss. The corresponding damped sinusoids are called natural modes. Same with the eigenmodes, the natural resonant frequencies and natural modes are independent of incident field. More importantly, the natural modes are special cases of the eigenmodes. At natural resonant frequencies, the integral equation kernels become singular, and the corresponding eigenmodes become natural modes [4]. The resonances of natural modes can be classified into internal resonances and external resonances [7]. The internal resonances are known as cavity resonances caused by the internal waves experiencing multiple internal reflections. The external resonances are caused by creeping waves propagating along the body surface with attenuation due to radiative loss [8]. Therefore, the internal resonant frequencies are purely real, while the external resonant frequencies are complex.

Unlike the SEM, a different approach called CMA is being used to find other kinds of modes and resonances in the real frequency domain. The CMA was initially defined by Garbacz [9] and was reformulated by Harrington and 
Mautz through diagonalizing the electric field integral equation (EFIE) operator for PEC bodies [6]. As the same as the eigenmodes and the natural modes, the characteristic modes are independent of incident field. However, differently from the eigenmodes and the natural modes, the characteristic values and characteristic currents of characteristic modes are both real, which facilitates their manipulation and interpretation. More importantly, the characteristic values of characteristic modes represent the ratio between the net stored power and the radiated power. Hence, the resonant behaviors can be predicted by the characteristic values. Same with the natural modes' resonances, the characteristic modes' resonances can also be classified into internal resonances and external resonances. When the characteristic value is zero, the corresponding mode is the external resonant mode. When the characteristic value tends to be infinite, the corresponding mode becomes the internal resonant mode [6].

The external resonances of natural modes are related to the resonant peaks of the scattering cross section [7], which means it can imply the maximum wave scattering. However, the characteristics of the electromagnetic power of natural modes for arbitrary PEC bodies are not involved in previous publications, only several canonical structures are investigated, such as the sphere. In this paper, we use the Poynting's theorem in complex frequency domain [10] to specify the characteristics of electromagnetic power of natural modes for arbitrary PEC bodies for the first time. We found that the natural modes of arbitrary PEC bodies are related to electromagnetic power resonances. More specifically, the net stored power of a natural mode is zero.

All of the three kinds of modes are independent of incident field, as introduced above. More importantly, they are all related to resonances. Therefore, it is essential to study the relationships between the three kinds of modes. Some efforts have been done in this aspect $[4,6,8,9,11-16]$.

In $[8,9]$, the relationship between the eigenvalues of eigenmodes and the characteristic values of characteristic modes was established. The relationship shows that the characteristic values of characteristic modes are the ratios between the imaginary parts and the real parts of the eigenvalues of eigenmodes. However, we found that the relationship is only valid in certain particular situations. More specifically, only when the surfaces of PEC bodies coincide with coordinate surfaces, such as the infinite cylinder and the sphere, the relationship is exactly accurate; otherwise, it is approximative. The eigenvalues and characteristic values of three typical structures are presented in Section 3 to demonstrate the conclusion.

In $[8,11,12,15,16]$, it was shown that the internal resonant frequencies of natural modes coincide with those of characteristic modes. Besides, Alroughani and Rezaiesarlak and Manteghi [11, 12] demonstrated that the external resonant frequencies of natural modes coincide with those of characteristic modes; that is, the external resonant frequencies of characteristic modes are the real part of the external resonant frequencies of natural modes. However, in [8], there is opposite viewpoint about this. According to careful investigations in this paper, we support the viewpoint in [8]. Moreover, besides resonant frequencies, after comparing the current distributions, phase distributions of current, and radiation patterns of the three kinds of modes, we find that there are corresponding relationships between the three kinds of modes. More specifically, there is a one-to-one corresponding relationship between the eigenmodes and the characteristic modes, as well as a one-to-many corresponding relationship between the eigenmodes and the natural modes. The corresponding relationships are not involved in previous publications.

\section{Definitions and Electromagnetic Power of Eigenmodes, Natural Modes, and Characteristic Modes}

To specify the corresponding relationships between eigenmodes, natural modes, and characteristic modes of PEC bodies, the definitions of the three kinds of modes are briefly reviewed in this section.

Consider one or more PEC bodies, which are defined by $S$, in an impressed electric field $\vec{E}^{\mathrm{i}}$. The impressed field induces an electric current distribution $\vec{J}$ on $S$ that radiates a scattered field $\vec{E}^{s}$. Applying the boundary conditions of tangential field on $S$ and considering that the scattered field can be expressed as $\vec{E}^{s}=-\mathbf{L}(\vec{J})$ and $\left[\vec{E}^{s}\right]_{\tan }=[-\mathbf{L}(\vec{J})]_{\tan }$ $=-\mathbf{Z}(\vec{J})$, the problem can be solved by EFIE [2]

$$
\mathrm{Z}(\vec{J}(\vec{r}, \omega))=\left[\vec{E}^{\mathrm{i}}(\vec{r}, \omega)\right]_{\tan },
$$

where the subscript tan denotes the tangential component. The operator $\mathbf{Z}$ is the tangential component of operator $\mathbf{L}$, and it is defined in [2]. $\vec{r}$ denotes the field point, and $\omega$ the angular frequency.

The induced electric current $\vec{J}$ on $S$ can be directly solved through the inverse of the operator $\mathbf{Z}$, that is, $\vec{J}(\vec{r}$, $\omega)=\mathbf{Z}^{-1} \vec{E}^{\mathrm{i}}(\vec{r}, \omega)$. Besides, various modal analysis methods can be used to obtain $\vec{J}$. For convenience, we define the symmetric product of two vector functions $\vec{A}$ and $\vec{B}$ in $\Omega$ as

$$
\langle\vec{A}, \vec{B}\rangle_{\Omega}=\int_{\Omega} \vec{A} \cdot \vec{B} d \Omega
$$

2.1. Eigenmodes. The eigenmodes are defined by the following standard eigenvalue equation $[3,6]$

$$
\mathbf{Z}\left(\vec{J}_{n}^{\mathrm{eig}}(\vec{r}, \omega)\right)=v_{n} \vec{J}_{n}^{\mathrm{eig}}(\vec{r}, \omega)
$$

In (3), $v_{n}$ are eigenvalues and $\vec{J}_{n}^{\text {eig }}$ are corresponding eigenmodes. It is clear from (3) that the eigenvalues and the eigenmodes obtained with (3) are independent of incident field, since operator $\mathbf{Z}$ is independent of incident field. Notice that both $v_{n}$ and $\vec{J}_{n}^{\text {eig }}$ are generally complex. $\vec{J}_{n}^{\text {eig }}$ is generally complex which indicates that $\vec{J}_{n}^{\text {eig }}$ on $S$ is not equiphase in 
general (except in some special cases, such as infinite circular cylinder and sphere). Considering that the tangential component of scattered field can be expressed as $\left[\vec{E}^{s}\right]_{\tan }=-\mathbf{Z}(\vec{J})$, we have

$$
\left[\vec{E}_{n}^{\mathrm{s}}\right]_{\tan }=-\mathbf{Z}\left(\vec{J}_{n}^{\mathrm{eig}}\right)=-v_{n} \vec{J}_{n}^{\mathrm{eig}} .
$$

Equation (4) indicates that the current and the tangential component of electric field of eigenmode have the same distribution on the surfaces of PEC bodies. Using (3) and (4) and considering the Poynting's theorem [1], the characteristics of electromagnetic power of eigenmodes can be obtained [17].

$v_{n}=\frac{\left\langle\left(\vec{J}_{n}^{\text {eig }}\right)^{*}, \mathbf{Z}\left(\vec{J}_{n}^{\mathrm{eig}}\right)\right\rangle_{S}}{\left\langle\left(\vec{J}_{n}^{\mathrm{eig}}\right)^{*}, \vec{J}_{n}^{\mathrm{eig}}\right\rangle_{S}}=\frac{P_{\mathrm{rad}}\left(\vec{J}_{n}^{\mathrm{eig}}\right)+j \omega\left[W_{m}\left(\vec{J}_{n}^{\mathrm{eig}}\right)-W_{e}\left(\vec{J}_{n}^{\mathrm{eig}}\right)\right]}{\left\langle\left(\vec{J}_{n}^{\mathrm{eig}}\right)^{*}, \vec{J}_{n}^{\mathrm{eig}}\right\rangle_{S}}$,

where $P_{\text {rad }}\left(\vec{J}_{n}^{\text {eig }}\right)$ and $W_{m}\left(\vec{J}_{n}^{\text {eig }}\right)-W_{e}\left(\vec{J}_{n}^{\text {eig }}\right)$ represent the radiated power and the net stored energy of the eigenmode, respectively. Notice that they are the functions of $\vec{J}_{n}^{\text {eig }}$. If $\left\langle\left(\vec{J}_{n}^{\text {eig }}\right)^{*}, \vec{J}_{n}^{\text {eig }}\right\rangle_{S}$ is normalized to unit, then

$$
v_{n}=P_{\text {rad }}\left(\vec{J}_{n}^{\mathrm{eig}}\right)+j \omega\left[W_{m}\left(\vec{J}_{n}^{\mathrm{eig}}\right)-W_{e}\left(\vec{J}_{n}^{\mathrm{eig}}\right)\right] .
$$

It can be observed from (6) that the real part and the imaginary part of $v_{n}$ represent the radiated power and the net stored power of the eigenmode, respectively. Equation (6) can be rewritten as

$$
\begin{aligned}
v_{n} & =\left|v_{n}\right| e^{j \tan ^{-1} \lambda_{n}^{\mathrm{eig}}}, \\
\lambda_{n}^{\mathrm{eig}} & =\frac{\omega\left[W_{m}\left(\vec{J}_{n}^{\mathrm{eig}}\right)-W_{e}\left(\vec{J}_{n}^{\mathrm{eig}}\right)\right]}{P_{\mathrm{rad}}\left(\vec{J}_{n}^{\mathrm{eig}}\right)} .
\end{aligned}
$$

Equation (7) implies the relationship between the eigenvalues of eigenmodes and the characteristic values of characteristic modes. The $\lambda_{n}^{\text {eig }}$ in (7) is very similar to the characteristic value $\lambda_{n}^{\text {cha }}$ of characteristic mode (defined in (24)). In Section 3, we will show that only when the surfaces of PEC bodies coincide with coordinate surfaces, the current distributions of eigenmodes and characteristic modes are identical; thus, the $\lambda_{n}^{\text {eig }}$ in (7) is identical to the characteristic value $\lambda_{n}^{\text {cha }}$ of characteristic mode. Otherwise, the $\lambda_{n}^{\text {eig }}$ in (7) is similar but not identical to the characteristic value $\lambda_{n}^{\text {cha }}$ of characteristic mode. Equation (7) is also presented in $[8,9]$; however, it is impertinently concluded that the $\lambda_{n}^{\text {eig }}$ in (7) is identical to the characteristic value $\lambda_{n}^{\text {cha }}$ of characteristic mode in any case.

It is obvious that $\lambda_{n}^{\text {eig }}$ represents the ratio between the net stored power and the radiated power. A mode with small $\lambda_{n}^{\text {eig }}$ indicates that the mode has weak net stored power and strong radiated power relatively. Therefore, it indicates that those $\lambda_{n}^{\text {eig }}$ of smallest magnitude are important for radiation and scattering problems. In general, modal significance (MS) is more convenient than $\lambda_{n}^{\text {eig }}$ to investigate the resonant behavior over a wide frequency band. It is defined as

$$
\mathrm{MS}=\frac{1}{\left|1+j \lambda_{n}^{\text {eig }}\right|}
$$

The MS transforms the $[-\infty,+\infty]$ range of $\lambda_{n}^{\text {eig }}$ into a much smaller range of $[0,1]$. We call those modes with $\lambda_{n}^{\text {eig }}>0$ inductive modes, those modes with $\lambda_{n}^{\text {eig }}<0$ capacitive modes, those modes with $\lambda_{n}^{\text {eig }}=0(\mathrm{MS}=1)$ external resonant modes, and those modes with $\mathrm{MS}=1 \rightarrow \infty$ (asymptotic behavior, MS $\rightarrow 0$ ) internal resonant modes.

It is noteworthy that when the eigenmode is resonant, the eigenmode does not generally satisfy the source-free condition, that is,

$$
\vec{E}_{n}^{s}(\vec{r}) \neq 0, \quad \vec{r} \in S
$$

This is different from natural modes. Furthermore, the eigenmodes hold the orthogonality [6].

$$
\left\langle\vec{J}_{m}^{\mathrm{eig}}, \mathbf{Z}\left(\vec{J}_{n}^{\mathrm{eig}}\right)\right\rangle_{S}=0, \quad m \neq n
$$

As previously mentioned, $\vec{J}_{m}^{\text {eig }}$ is generally complex, which means $\vec{J}_{m}^{\text {eig }} \neq\left(\vec{J}_{m}^{\text {eig }}\right)^{*}$. Therefore, (10) indicates that eigenmodes do not hold the electromagnetic power orthogonality in general. This makes it different from characteristic modes.

Applying the orthogonality, any induced electric current $\vec{J}$ can be written as the linear combinations of the eigenmodes.

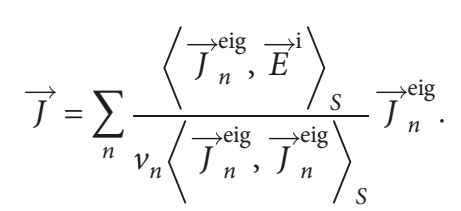

This method is known as EEM. More details can be found in [3].

2.2. Natural Modes. A natural mode is a field that can exist in the absence of impressed sources [7], that is, $\vec{E}^{i}=0$ in (1) and

$$
\mathbf{Z}\left(\vec{J}_{n}^{\text {nat }}\left(\vec{r}, \omega_{c}\right)\right)=0=0 \cdot \vec{J}_{n}^{\text {nat }}\left(\vec{r}, \omega_{c}\right) .
$$

Comparing (3) with (12), it is found that a natural mode is a special eigenmode whose eigenvalue is zero. Equation (12) indicates that $\mathbf{Z}$ is a singular operator. This generally occurs at some discrete complex frequencies, known as natural frequencies $\omega_{c}=\omega^{\prime}+j \omega^{\prime \prime}$. Here, $\omega^{\prime}$ is the natural resonant frequency of a given natural mode, and $\omega^{\prime \prime}$ is the damping factor due to radiative loss [4]. It is called internal resonance 
when $\omega^{\prime \prime}=0$ and external resonance when $\omega^{\prime \prime} \neq 0$. The natural modes obtained with (12) are independent of incident field.

One of the critical characters of natural modes is that the natural modes can exist in the absence of incident fields. It is clear from the definition of natural modes; that is, the natural modes are calculated by making $\vec{E}^{\mathrm{i}}=0$, which is not only a mathematics method but also brings significant physical meanings. More specifically, thanks to making $\vec{E}^{\mathrm{i}}=0$, the natural modes can satisfy the boundary conditions by itself. For example, the cavity modes of PEC-enclosed cavity are internal resonant modes of natural modes. Once those modes are excited in one way, they can hold the self-oscillations without loss forever. Concerning the open problem, external resonant modes of natural modes can still hold the self-oscillations, which unfortunately are damped by radiative loss [7].

The characteristics of the electromagnetic power of natural modes for arbitrary PEC bodies are not involved in previous publications, only several canonical structures are investigated, such as the sphere. Here, we use the Poynting's theorem in complex frequency domain [10] to specify the characteristics of the electromagnetic power of natural modes of arbitrary PEC bodies, to our best knowledge, for the first time.

The Maxwell's equations in the complex frequency domain are

$$
\begin{gathered}
\nabla \times \vec{E}=-j \omega_{c} \mu \vec{H}, \\
\nabla \times \vec{H}=\vec{J}+j \omega_{c} \varepsilon \vec{E} .
\end{gathered}
$$

It follows from (13) that

$$
\begin{aligned}
(\nabla \times \vec{E}) \cdot \vec{H}^{*} & =-j \omega_{c} \mu \vec{H} \cdot \vec{H}^{*}, \\
(\nabla \times \vec{H})^{*} \cdot \vec{E} & =\vec{J}^{*} \cdot \vec{E}-j \omega_{c}{ }^{*} \varepsilon \vec{E}^{*} \cdot \vec{E}
\end{aligned}
$$

Considering the following vector identity,

$$
\nabla \cdot\left(\vec{E} \times \vec{H}^{*}\right)=(\nabla \times \vec{E}) \cdot \vec{H}^{*}-\left(\nabla \times \vec{H}^{*}\right) \cdot \vec{E}
$$

results in

$$
-\vec{J}^{*} \cdot \vec{E}=\nabla \cdot\left(\vec{E} \times \vec{H}^{*}\right)+j\left(\omega_{c} \mu \vec{H} \cdot \vec{H}^{*}-\omega_{c}^{*} \varepsilon \vec{E}^{*} \cdot \vec{E}\right) .
$$

Substituting $\omega_{c}=\omega^{\prime}+j \omega^{\prime \prime}$ into (16), we have

$$
\begin{array}{r}
-\vec{J}^{*} \cdot \vec{E}=\left[\nabla \cdot\left(\vec{E} \times \vec{H}^{*}\right)\right. \\
\left.-\omega^{\prime \prime}\left(\mu \vec{H} \cdot \vec{H}^{*}+\varepsilon \vec{E}^{*} \cdot \vec{E}\right)\right] \\
+j \omega^{\prime}\left(\mu \vec{H} \cdot \vec{H}^{*}-\varepsilon \vec{E}^{*} \cdot \vec{E}\right) .
\end{array}
$$

Taking the integration of (17) over the region $V_{\infty}$ bounded by $\partial V_{\infty}$ gives

$$
P_{\text {sup }}=\left[-2 \omega^{\prime \prime}\left(W_{m}+W_{e}\right)+P_{\text {rad }}\right]+j 2 \omega^{\prime}\left(W_{m}-W_{e}\right),
$$

where

$$
\begin{aligned}
P_{\text {sup }} & =-\frac{1}{2}\left\langle\vec{J}^{*}, \vec{E}\right\rangle_{s} \\
W_{m} & =\frac{1}{4} \mu\left\langle\vec{H}, \vec{H}^{*}\right\rangle_{V \infty}, \quad \times \vec{H}^{*} \cdot d \vec{S} . \\
W_{e} & =\frac{1}{4} \varepsilon\left\langle\vec{E}, \vec{E}^{*}\right\rangle_{V \infty}, \\
P_{\text {rad }} & =-\frac{1}{2} \iint_{\partial V_{\infty}} \vec{E}
\end{aligned}
$$

In the above equations, $\partial V_{\infty}$ represents the sphere at infinity, and $V_{\infty}$ represents the volume surrounded by $\partial$ $V_{\infty}$. Equation (18) is called the Poynting's theorem in complex frequency domain. Notice that if $\omega^{\prime \prime}=0,(18)$ reduces to time-harmonic Poynting's theorem as follows [1]:

$$
P_{\text {sup }}=P_{\text {rad }}+j 2 \omega^{\prime}\left(W_{m}-W_{e}\right) .
$$

A natural mode means that $[\vec{E}(\vec{r})]_{\tan }=0$ where $\vec{r}$ represents the source region. Thus, $P_{\text {sup }}=-1 / 2\left\langle\vec{J}^{*}, \vec{E}\right\rangle_{S}=0$, meanwhile $W_{e}=1 / 4 \varepsilon\left\langle\vec{E}, \vec{E}^{*}\right\rangle_{V \infty} \neq 0$. Therefore, concerning natural modes, we have

$$
\begin{aligned}
-2 \omega^{\prime \prime}\left(W_{m}+W_{e}\right)+P_{\text {rad }} & =0, \\
W_{m}-W_{e} & =0 .
\end{aligned}
$$

Therefore, the difference between the electric and magnetic energy of a natural mode is zero, and this is why we called the natural mode as the natural resonant mode. However, it must be emphasized that there is a significant difference between the resonances of natural modes and the resonances of eigenmodes and characteristic modes. The resonant natural modes can hold its resonant state by itself, whereas the eigenmodes and characteristic modes require impressed field to maintain its resonant state. It is because the natural modes can exist in the absence of impressed sources, whereas the eigenmodes and characteristic modes cannot. Because of the absence of impressed sources, the fields and currents of natural modes must decay with time due to radiative loss, which can be demonstrated mathematically using

$$
\vec{F}(\vec{r}, t)=\vec{F}(\vec{r}) e^{j \omega_{c} t}=\vec{F}(\vec{r}) e^{-\omega^{\prime \prime} t} e^{j \omega^{\prime} t},
$$

in which $\vec{F}(\vec{r}, t)$ represents field or current, which is the function of position and time. $\overrightarrow{\dot{F}}(\vec{r})$ is the corresponding phasor which is only the function of position. $\omega_{c}=\omega^{\prime}+j \omega^{\prime \prime}$ is the complex natural resonant frequency. It can be clearly observed from (22) that the fields and currents of natural modes decay with time (except $\omega^{\prime \prime}=0$ which is corresponding to internal resonance). 
However, eigenmodes and characteristic modes cannot exist in the absence of impressed sources since the frequency is real. And eigenmodes and characteristic modes both are time-harmonic fields. In fact, each eigenmode or characteristic mode corresponds to a distribution of incident field, which is the key point to excite specific modes [18]. For example, if we consider the modes of PEC bodies, we denote the current of the $n$th modes as $\vec{J}_{n}$, and the modal fields of the $n$th modes as $\left(\vec{E}_{n}, \vec{H}_{n}\right)$. Following the boundary conditions, the incident field that corresponds to the $n$th modes satisfies

$$
\begin{aligned}
& \widehat{n} \times\left[\vec{E}_{n}^{\text {inc }}(\vec{r})+\vec{E}_{n}(\vec{r})\right]=0, \quad \vec{r} \in \partial V, \\
& \hat{n} \times\left[\vec{H}_{n}^{\text {inc }}(\vec{r})+\vec{H}_{n}(\vec{r})\right]=\vec{J}(\vec{r}), \quad \vec{r} \in \partial V,
\end{aligned}
$$

where $\partial V$ represents the surfaces of PEC bodies and $\widehat{n}$ represents the normal vectors of $\partial V \cdot\left(\vec{E}_{n}^{\text {inc }}, \vec{H}_{n}^{\text {inc }}\right)$ which can completely excite the $n$th modes.

In a word, the natural modes can satisfy the boundary conditions by itself, while the eigenmodes and the characteristic modes cannot. Thus, the natural modes can exist in the absence of impressed sources, whereas the eigenmodes and the characteristic modes cannot. Therefore, the resonance of natural modes is different from the resonances of eigenmodes and characteristic modes.

2.3. Characteristic Modes. The characteristic modes are defined by the following generalized eigenvalue equation [6]:

$$
\mathbf{X}\left(\vec{J}_{n}^{\text {cha }}(\vec{r}, \omega)\right)=\lambda_{n}^{\text {cha }} \mathbf{R}\left(\vec{J}_{n}^{\text {cha }}(\vec{r}, \omega)\right),
$$

where $\mathbf{X}$ and $\mathbf{R}$ are the imaginary and real parts of $\mathbf{Z}$. In (24), $\lambda_{n}^{\text {cha }}$ are characteristic values and $\vec{J}_{n}^{\text {cha }}$ are corresponding characteristic modes. The characteristic values and characteristic modes obtained with (24) are independent of incident field. It is important to note that both $\lambda_{n}^{\text {cha }}$ and $\vec{J}_{n}^{\text {cha }}$ are real. $\vec{J}_{n}^{\text {cha }}$ is real which indicates that $\vec{J}_{n}^{\text {cha }}$ on $S$ is equiphase. This is different from eigenmodes and natural modes, which are generally complex.

Considering Poynting's theorem [1], we have

$$
\begin{aligned}
& \left\langle\vec{J}_{n}^{\text {cha }}, \mathbf{R}\left(\vec{J}_{n}^{\text {cha }}\right)\right\rangle_{S}=P_{\text {rad }}\left(\vec{J}_{n}^{\text {cha }}\right), \\
& \left\langle\vec{J}_{n}^{\text {cha }}, \mathbf{X}\left(\vec{J}_{n}^{\text {cha }}\right)\right\rangle_{S}=\omega\left[W_{m}\left(\vec{J}_{n}^{\text {cha }}\right)-W_{e}\left(\vec{J}_{n}^{\text {cha }}\right)\right],
\end{aligned}
$$

where $P_{\text {rad }}\left(\vec{J}_{n}^{\text {cha }}\right)$ and $W_{m}\left(\vec{J}_{n}^{\text {cha }}\right)-W_{e}\left(\vec{J}_{n}^{\text {cha }}\right)$ represent the radiated power and the net stored energy of corresponding mode, respectively. Notice that they are the functions of $\vec{J}_{n}^{\text {cha }}$. Using (24) and (25), we have

$$
\lambda_{n}^{\text {cha }}=\frac{\left\langle\vec{J}_{n}^{\text {cha }}, \mathbf{X}\left(\vec{J}_{n}^{\text {cha }}\right)\right\rangle_{S}}{\left\langle\vec{J}_{n}^{\text {cha }}, \mathbf{R}\left(\vec{J}_{n}^{\text {cha }}\right)\right\rangle_{S}}=\frac{\omega\left[W_{m}\left(\vec{J}_{n}^{\text {cha }}\right)-W_{e}\left(\vec{J}_{n}^{\text {cha }}\right)\right]}{P_{\text {rad }}\left(\vec{J}_{n}^{\text {cha }}\right)} .
$$

It is noteworthy that $\lambda_{n}^{\text {cha }}$ in (26) is not identical to $\lambda_{n}^{\text {eig }}$ in (7), because $\vec{J}_{n}^{\text {cha }}$ is not identical to $\vec{J}_{n}^{\text {eig }}$ generally. We will demonstrate it using numerical results in Section 3.

The MS of characteristic modes also can be defined as (4). From (26), it is obvious that $\lambda_{n}^{\text {cha }}$ represents the ratio between the net stored power and the radiated power. We call those modes with $\lambda_{n}^{\text {cha }}>0$ inductive modes, those modes with $\lambda_{n}^{\text {cha }}<0$ capacitive modes, those modes with $\lambda_{n}^{\text {cha }}=0(\mathrm{MS}=1)$ external resonant modes, and those modes with $\lambda_{n}^{\text {cha }} \rightarrow \infty \quad$ (asymptotic behavior, MS $\rightarrow 0$ ) internal resonant modes [6]. Notice that when the characteristic mode is external resonant, it does not generally satisfy the source-free condition. This is quite different with natural modes.

If the radiated power is normalized to unit, the characteristic modes hold the following orthogonality [6].

$$
\begin{aligned}
& \left\langle\vec{J}_{m}^{\text {cha }}, \mathbf{R}\left(\vec{J}_{n}^{\text {cha }}\right)\right\rangle_{S}=\delta_{m n}, \\
& \left\langle\vec{J}_{m}^{\text {cha }}, \mathbf{X}\left(\vec{J}_{n}^{\text {cha }}\right)\right\rangle_{S}=\lambda_{m} \delta_{m n}, \\
& \left\langle\vec{J}_{m}^{\text {cha }}, \mathbf{Z}\left(\vec{J}_{n}^{\text {cha }}\right)\right\rangle_{S}=\left(1+j \lambda_{m}\right) \delta_{m n},
\end{aligned}
$$

where $\delta_{m n}$ is the Kronecker delta ( 0 if $m \neq n$ and 1 if $m=n$ ). In view of currents is real, (27) represents the electromagnetic power orthogonality. This makes it different from eigenmodes. Applying the orthogonality, any induced electric current $\vec{J}$ can be written as the linear combinations of the characteristic modes [6].

$$
\vec{J}=\sum_{n} \frac{\left\langle\vec{J}_{n}^{\text {cha }}, \vec{E}^{i}\right\rangle_{S}}{1+j \lambda_{n}^{\text {cha }}} \vec{J}_{n}^{\text {cha }} .
$$

To make the relationship and distinction between the three kinds of modes clear, we summarize the modal definitions and properties in Table 1.

\section{Modes of Three Typical Structures}

In this section, the eigenmodes, natural modes, and characteristic modes of three typical PEC structures are investigated. These structures include the PEC infinite circular cylinder, the PEC sphere, and the PEC rectangular plate: (1) The PEC infinite circular cylinder is an example of 
TABLE 1: Summarization of modal definitions and properties.

\begin{tabular}{|c|c|c|c|}
\hline Mode & igenmode & Natural mode & Characteristic mode \\
\hline Current & $\vec{J}_{n}^{\text {eig }}$ & $\vec{J}_{n}^{\text {nat }}$ & $\vec{J}_{n}^{\text {cha }}$ \\
\hline Definition & $\mathbf{Z}\left(\vec{J}_{n}^{\mathrm{eig}}(\vec{r}, \omega)\right)=v_{n} \vec{J}_{n}^{\mathrm{eig}}(\vec{r}, \omega)$ & $\mathbf{Z}\left(\vec{J}_{n}^{\text {nat }}\left(\vec{r}, \omega_{c}\right)\right)=0=0 \cdot \vec{J}_{n}^{\text {nat }}\left(\vec{r}, \omega_{c}\right)$ & $\mathbf{X}\left(\vec{J}_{n}^{\text {cha }}(\vec{r}, \omega)\right)=\lambda_{n}^{\text {cha }} \mathbf{R}\left(\vec{J}_{n}^{\text {cha }}(\vec{r}, \omega)\right)$ \\
\hline Eigen & $v_{n}=P_{\mathrm{rad}}+j \omega\left(W_{m}-W_{e}\right)$ & 0 & $\lambda_{n}^{\mathrm{cha}}=\omega\left(W_{m}-W_{e}\right) / P_{\mathrm{rad}}$ \\
\hline Main & \multicolumn{3}{|c|}{$\begin{array}{l}\text { (1) } v_{n} \text { is generally a complex number, while } \lambda_{n} \text { is a real number. } \\
\text { (2) } \vec{J}_{n}^{\text {eig }} \text { and } \vec{J}_{n}^{\text {nat }} \text { are generally complex, while } \vec{J}_{n}^{\text {cha }} \text { is real. } \\
\text { (3) Linked to (2), } J_{n}^{\text {eig }} \text { and } \vec{J}_{n}^{\text {nat }} \text { are not equiphase on the surface of PEC bodies generally, while } \vec{J}_{n}^{\text {cha }} \text { is always } \\
\text { equiphase. } \\
\text { (4) } \lambda_{n}^{\text {cha }} \text { is similar but not identical to the ratios between the real parts and the imaginary parts of } v_{n} \text { in general. } \\
\text { (5) The natural modes can exist in the absence of impressed fields, while the eigenmodes and characteristic } \\
\text { modes cannot. }\end{array}$} \\
\hline
\end{tabular}

two-dimensional canonical structures; (2) The PEC sphere is an example of three-dimensional canonical structures; (3) The PEC rectangular plate is an example of noncanonical structures. The corresponding relationships between the three kinds of modes are illustrated through these three structures.

3.1. PEC Infinite Circular Cylinder. In this section, the closed forms of the eigenmodes, natural modes, and characteristic modes of a PEC infinite circular cylinder are investigated. We also present the comparisons about resonant frequencies and current distributions of the three kinds of modes.

The eigenmodes of the PEC infinite circular cylinder can be classified into $\mathrm{TM}_{z}$ and $\mathrm{TE}_{z}$ modes. The eigenvalues of $\mathrm{T}$ $\mathrm{M}_{z}$ and $\mathrm{TE}_{z}$ modes are [17]

$$
\begin{aligned}
& v_{n}^{\mathrm{TM}}=\frac{\eta \pi k a}{2} J_{n}(k a) H_{n}^{(2)}(k a), \\
& v_{n}^{\mathrm{TE}}=\frac{\eta \pi k a}{2} J_{n}^{\prime}(k a) H_{n}^{\prime(2)}(k a),
\end{aligned}
$$

where $a$ is the cylinder's radius, $J_{n}(x)$ is the Bessel functions of first kind, $H_{n}^{(2)}(x)$ is the Hankel functions of second kind, $J_{n}^{\prime}(x)$ is the derivative of $J_{n}(x), H_{n}^{\prime(2)}(x)$ is the derivative of $H_{n}{ }^{(2)}(x)$ [19], $n$ is the azimuthal mode order, $k$ is the wavenumber, and $\eta$ is the intrinsic impedance.

As we demonstrated in Section 2, a natural mode is a special eigenmode whose eigenvalue is zero. From (29) and (30), it is found that the internal resonant frequencies of natural modes can be obtained with $J_{n}(k a)=0$ and $J_{n}^{\prime}(k a)=0$, and the external resonant frequencies of natural modes can be obtained with $H_{n}{ }^{(2)}(k a)=0$ and $H_{n}^{\prime(2)}(k a)=0$. Therefore, an eigenmode can be linked to several natural modes because there are several roots of $B_{n}(x)=0$ [1], where $B_{n}(x)$ represents arbitrary Bessel functions.
The currents of eigenmodes are [17]

$$
\begin{aligned}
& \vec{J}_{n}^{\text {eig,TM }}=\widehat{z}\left\{\begin{array}{c}
\cos n \varphi \\
\sin n \varphi
\end{array}\right\}, \\
& \vec{J}_{n}^{\text {eig,TE }}=\widehat{\varphi}\left\{\begin{array}{c}
\cos n \varphi \\
\sin n \varphi
\end{array}\right\},
\end{aligned}
$$

where $\cos n \varphi$ represents even mode and $\sin n \varphi$ represents odd mode. Considering (31) and (32), we can find that the current distributions of eigenmodes are independent of frequency; therefore, the current distributions of eigenmodes and natural modes are identical. In addition, the currents are equiphase on $S$.

The closed forms of characteristic values of characteristic modes are [9]

$$
\begin{aligned}
& \lambda_{n}^{\mathrm{cha}, \mathrm{TM}}=-\frac{Y_{n}(k a)}{J_{n}(k a)}, \\
& \lambda_{n}^{\mathrm{cha}, \mathrm{TE}}=-\frac{Y_{n}^{\prime}(k a)}{J_{n}^{\prime}(k a)},
\end{aligned}
$$

where $Y_{n}(x)$ is the Bessel functions of second kind and $Y_{n}^{\prime}(x)$ is the derivative of $Y_{n}(x)$.

From (33) and (34), it is found that the internal resonant frequencies of characteristic modes can be obtained with $J_{n}$ $(k a)=0$ and $J_{n}^{\prime}(k a)=0$, and the external resonant frequencies of characteristic modes can be obtained with $Y_{n}(k a)=$ 0 and $Y_{n}^{\prime}(k a)=0$. Considering the properties of $J_{n}(x)$ and $Y_{n}(x)$ [19], both the internal and external resonant frequencies of characteristic modes are real. This is different from natural modes, whose external resonant frequencies are complex. Considering (29), (30), (33), and (34), it is obvious that both the natural modes and characteristic modes indicate the same internal resonant frequencies, that is, $J_{n}(k a)=0$ or $J_{n}^{\prime}(k a)$. However, careful investigations in this section show that the external resonant frequencies of characteristic modes do not coincide with the real part of the 


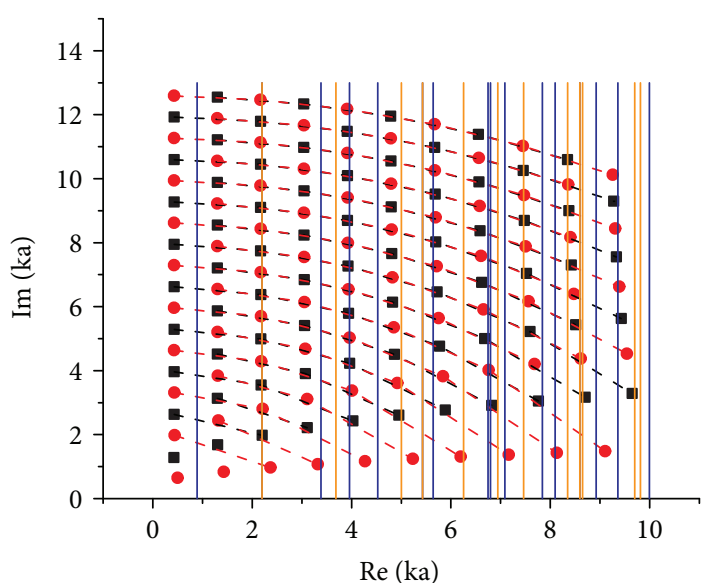

- TM natural resonances - TM characteristic resonances

- TE natural resonances — TE characteristic resonances

Figure 1: The external resonant frequencies of natural modes and characteristic modes of cylinder.

external resonant frequencies of natural modes. We calculate those external resonant frequencies whose $\operatorname{Re}(k a)<10$ as shown in Figure 1. From Figure 1, it is obvious that the external resonant frequencies of characteristic modes do not coincide with the real part of the external resonant frequencies of natural modes. The conclusion agrees with [8] and disagrees with [11].

In addition, the closed forms of characteristic mode currents are [9]

$$
\begin{aligned}
& \vec{J}_{n}^{\mathrm{cha}, \mathrm{TM}}=\widehat{z} \frac{\sqrt{\varepsilon_{n}}}{2 \pi a J_{n}(k a)}\left\{\begin{array}{c}
\cos n \varphi \\
\sin n \varphi
\end{array}\right\}, \\
& \vec{J}_{n}^{\mathrm{cha}, \mathrm{TE}}=\widehat{\varphi} \frac{\sqrt{\varepsilon_{n}}}{2 \pi a J_{n}^{\prime}(k a)}\left\{\begin{array}{l}
\cos n \varphi \\
\sin n \varphi
\end{array}\right\},
\end{aligned}
$$

where

$$
\varepsilon_{n}= \begin{cases}1, & n=0 \\ 2, & n \neq 0\end{cases}
$$

The coefficients $\sqrt{\varepsilon_{n}} / 2 \pi a J_{n}(k a)$ and $\sqrt{\varepsilon_{n}} / 2 \pi a J_{n}^{\prime}(k a)$ are used to normalize the radiated power to unit, and they do not affect the current distributions. If we ignore the coefficients, comparing (31), (32), (35), and (36), we can find that the current distributions of eigenmodes, natural modes, and the characteristic modes are identical.

3.2. PEC Sphere. In this section, we investigate the closed forms of the eigenmodes, natural modes, and characteristic modes of a PEC sphere. The comparisons about resonant frequencies and current distributions of the three kinds of modes are also presented.

The eigenmodes of the PEC sphere can be classified as $\mathrm{TM}_{r}$ and $\mathrm{TE}_{r}$ modes. The closed forms of eigenmodes of the PEC sphere (defined in (3)) do not exist in previous publications. Therefore, we firstly derive the closed forms of eigenmodes. Here, we only give the results of the closed forms of eigenmodes, and the specific derivations are presented in the Appendix for brevity.

The eigenvalues and currents of eigenmodes are

$$
\begin{aligned}
\vec{J}_{m n}^{\text {eig,TM }}=C_{\mathrm{EM}}^{\mathrm{TM}} & {\left[\widehat{\theta} \frac{\partial P_{n}^{m}(\cos \theta)}{\partial \theta}\left\{\begin{array}{c}
\cos m \phi \\
\sin m \phi
\end{array}\right\}\right.} \\
& \left.+\widehat{\phi} \frac{m P_{n}^{m}(\cos \theta)}{\sin \theta}\left\{\begin{array}{c}
-\sin m \phi \\
\cos m \phi
\end{array}\right\}\right],
\end{aligned}
$$

$$
\begin{aligned}
J_{m n}^{\mathrm{eig}, \mathrm{TE}}=C_{\mathrm{EM}}^{\mathrm{TE}}[ & -\hat{\theta} \frac{m P_{n}^{m}(\cos \theta)}{\sin \theta}\left\{\begin{array}{c}
-\sin m \phi \\
\cos m \phi
\end{array}\right\} \\
& \left.+\widehat{\phi} \frac{\partial P_{n}^{m}(\cos \theta)}{\partial \theta}\left\{\begin{array}{c}
\cos m \phi \\
\sin m \phi
\end{array}\right\}\right],
\end{aligned}
$$

$$
v_{m n}^{\mathrm{TM}}=-\frac{(1 / j \omega \varepsilon)\left[\left(\partial \widehat{J}_{n}(k r) / \partial r\right)\left(\partial \widehat{H}_{n}^{(2)}(k r) / \partial r\right)\right]_{r=a}}{\left.\left.\left(\partial \widehat{J}_{n}(k r) / \partial r\right)\right|_{r=a}\left(\widehat{H}_{n}^{(2)}(k a)-\widehat{J}_{n}(k a)\right)\left(\partial \widehat{H}_{n}^{(2)}(k r) / \partial r\right)\right|_{r=a}},
$$

$v_{m n}^{\mathrm{TE}}=j \omega \mu \frac{\widehat{J}_{n}(k a) \widehat{H}_{n}^{(2)}(k a)}{\left[\left(\widehat{J}_{n}(k a)\left(\partial \widehat{H}_{n}^{(2)}(k r) / \partial r\right)\right)-\left(\widehat{H}_{n}^{(2)}(k a)\right)\left(\partial \widehat{J}_{n}(k r) / \partial r\right)\right]_{r=a}}$,

where

$$
\begin{gathered}
C_{\mathrm{EM}}^{\mathrm{TM}}=\frac{b_{n} \widehat{H}_{n}^{(2)}(k a)-a_{n} \widehat{J}_{n}(k a)}{\mu a}, \\
C_{\mathrm{EM}}^{\mathrm{TE}}=\frac{\left[b_{n}\left(\partial \widehat{H}_{n}^{(2)}(k r) / \partial r\right)-a_{n}\left(\partial \widehat{J}_{n}(k r) / \partial r\right)\right]_{r=a}}{j \omega \mu \varepsilon a},
\end{gathered}
$$

in which $a$ is the sphere's radius, $\widehat{J}_{n}(x)$ is the Riccati-Bessel functions of first kind, and $\widehat{H}_{n}^{(2)}(x)$ is the Riccati-Hankel functions of second kind. $\cos m \varphi$ represents even mode, and $\sin m \varphi$ represents odd mode. As we demonstrated in Section 2, a natural mode is a special eigenmode whose eigenvalue is zero. From and, it is found that the internal resonant frequencies of natural modes can be obtained with $\widehat{J}_{n}(k a)=0$ and $\widehat{H}_{n}^{(2)}(k a)=0$, and the external resonant frequencies of natural modes can be obtained with $\widehat{H}_{n}^{(2)}(k a)=0$ and $\widehat{H}_{n}^{\prime}(2)(k a)=0$.

From (38) and (39), it can be observed that the current distributions of eigenmodes are independent of frequency. Hence, the current distributions of eigenmodes and natural modes are identical. In addition, the currents are equiphase on $S$. 
The closed forms of characteristic values of characteristic modes are [9]

$$
\begin{aligned}
\lambda_{n}^{\mathrm{TM}} & =\frac{\widehat{Y}_{n}^{\prime}(k a)}{\widehat{J}_{n}^{\prime}(k a)}, \\
\lambda_{n}^{\mathrm{TE}} & =-\frac{\widehat{Y}_{n}(k a)}{\widehat{J}_{n}(k a)},
\end{aligned}
$$

where $\widehat{Y}_{n}(x)$ is the Riccati-Bessel functions of second kind and $Y_{n}^{\prime}(x)=0$ is the derivative of $Y_{n}(x)$ [19].

From (43) and (44), it is found that the internal resonant frequencies of characteristic modes can be obtained with $\widehat{J}_{n}$ $(k a)=0$ and $J_{n}^{\prime}(k a)=0$, and the external resonant frequencies of characteristic modes can be obtained with $\widehat{Y}_{n}(x)=0$ and $Y_{n}^{\prime}(x)=0$. Considering the properties of $\widehat{J}_{n}(x)$ and $\widehat{Y}_{n}(x)$ [19], both the internal and external resonant frequencies of characteristic modes are real. This is different from natural modes, whose external resonant frequencies are complex. Considering (40), (41), (43), and (44), it is obvious that both the natural modes and characteristic modes indicate the same internal resonant frequencies, that is, $\widehat{J}_{n}(k a)=0$ and $\widehat{J}_{n}^{\prime}(k a)=0$. However, careful investigations in this section show that the external resonant frequencies of characteristic modes do not coincide with the real part of the external resonant frequencies of natural modes. We calculate those external resonant frequencies whose $\operatorname{Re}(k a)<10$ as shown in Figure 2. From Figure 2, it is obvious that the external resonant frequencies of characteristic modes do not coincide with the real part of the external resonant frequencies of natural modes.

In addition, the closed forms of characteristic mode currents are [9]

$$
\begin{aligned}
& \vec{J}_{m n}^{\mathrm{cha}, \mathrm{TM}}=C_{\mathrm{CM}}^{\mathrm{TM}}[ \widehat{\theta} \frac{\partial P_{n}^{m}(\cos \theta)}{\partial \theta}\left\{\begin{array}{c}
\cos m \phi \\
\sin m \phi
\end{array}\right\} \\
&\left.+\widehat{\phi} \frac{m P_{n}^{m}(\cos \theta)}{\sin \theta}\left\{\begin{array}{r}
-\sin m \phi \\
\cos m \phi
\end{array}\right\}\right], \\
& \vec{J}_{m n}^{\mathrm{cha}, \mathrm{TE}}=C_{\mathrm{CM}}^{\mathrm{TE}}[-\widehat{\theta} \frac{m P_{n}^{m}(\cos \theta)}{\sin \theta}\left\{\begin{array}{c}
-\sin m \phi \\
\cos m \phi
\end{array}\right\} \\
&\left.+\widehat{\phi} \frac{\partial P_{n}^{m}(\cos \theta)}{\partial \theta}\left\{\begin{array}{c}
\cos m \phi \\
\sin m \phi
\end{array}\right\}\right],
\end{aligned}
$$

where

$$
\begin{aligned}
C_{\mathrm{CM}}^{\mathrm{TM}} & =\frac{1}{k \sqrt{Z_{0}}} \frac{1}{\left.a^{2}\left[k r \cdot j_{n}(k r)\right]^{\prime}\right|_{r=a}} \sqrt{\frac{\varepsilon_{m}}{4 \pi} \frac{2 n+1}{n(n+1)} \frac{(n-m) !}{(n+m) !}}, \\
C_{\mathrm{CM}}^{\mathrm{TE}} & =\frac{1}{k \sqrt{Z_{0}}} \frac{1}{a^{2} j_{n}(k a)} \sqrt{\frac{\varepsilon_{m}}{4 \pi} \frac{2 n+1}{n(n+1)} \frac{(n-m) !}{(n+m) !}} .
\end{aligned}
$$

The coefficients $C_{\mathrm{CM}}^{\mathrm{TM}}$ and $C_{\mathrm{CM}}^{\mathrm{TE}}$ are used to normalize the radiated power to unit, and they do not affect the current

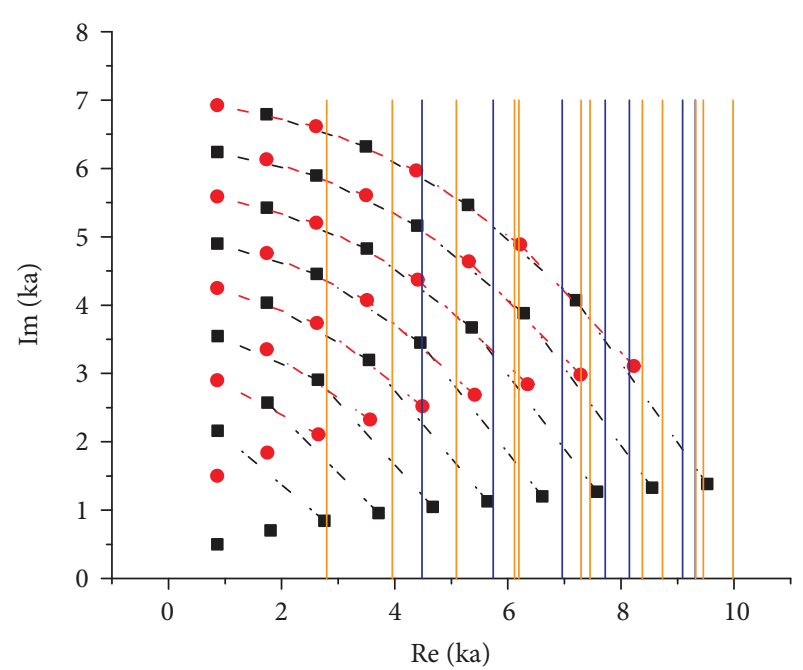

- TM natural resonances — TM characteristic resonances

- TE natural resonances — TE characteristic resonances

FIgURE 2: The external resonant frequencies of natural modes and characteristic modes of sphere.

distributions. If we ignore the coefficients, comparing, (38), (39), (45), and (46), it is found that the current distributions of eigenmodes, natural modes, and characteristic modes are identical.

Concerning the PEC infinite circular cylinder and sphere, it can be observed that

(1) there is a one-to-one corresponding relationship between the eigenmodes and the characteristic modes and a one-to-many corresponding relationship between the eigenmodes and the natural modes (The one-to-many corresponding relationship between the eigenmodes and the natural modes could be interpreted mathematically using the properties of zero of Bessel functions; besides, it could be explained physically in terms the wave mechanism that results in natural modes as explained in $[20,21]$. The current distributions of the three kinds of modes are identi$\mathrm{cal}$; all of them are equiphase on the surfaces of PEC bodies);

(2) the resonant frequencies of eigenmodes coincide with those of characteristic modes, as a result of identical current distributions;

(3) the internal resonant frequencies of natural modes coincide with those of eigenmodes and characteristic modes, whereas the external resonant frequencies of natural modes do not coincide with those of eigenmodes and characteristic modes.

3.3. PEC Rectangular Plate. As the last example, we consider a PEC rectangular plate of width $W=1$ meter and length $L=$ 1.1 meter as an example of noncanonical structures. The natural modes of the rectangular plate are presented in [22]. The eigenmodes and the characteristic modes are calculated using MoM in this section. The basis functions and test functions 


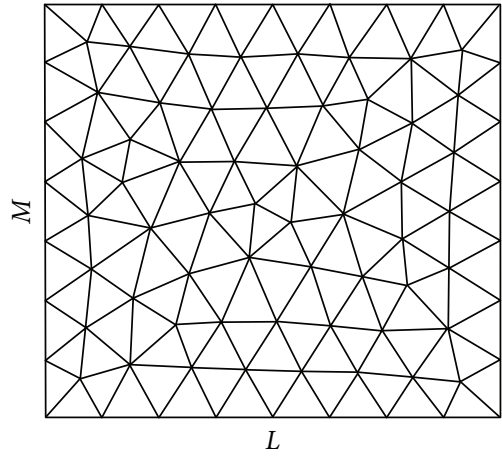

(a)

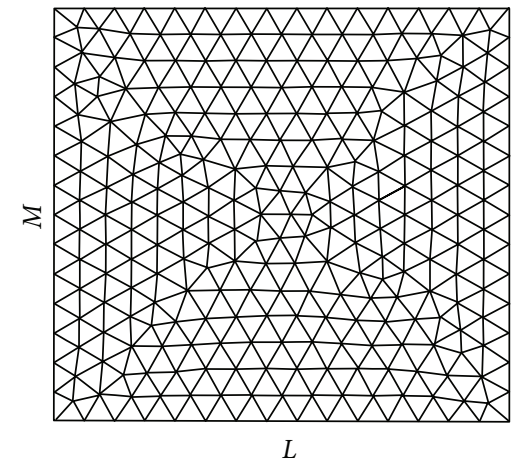

(b)

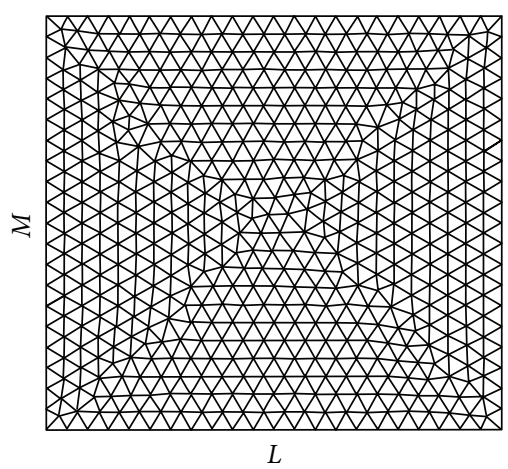

(c)

Figure 3: Three different meshes of the rectangular plate. (a) Mesh 1. (b) Mesh 2. (c) Mesh 3.

both are RWG functions [23]. The impedance matrix calculation is based on the method presented in [24]. The accuracy of the impedance matrix calculation depends on the singularity treatment in the calculation of diagonal and near-diagonal elements. In this paper, the singularity treatment is based on the method described in [25], which is proved to be very effective. To ensure that our numerical results are not influenced by the mesh density, we considered three different meshes as shown in Figure 3. The average length of the edges of the three meshes are $\lambda_{\min } / 10, \lambda_{\min } / 20$, and $\lambda_{\min } / 30$, respectively. The $\lambda_{\min }$ represents the wavelength corresponding to the highest frequency.

Using the three different meshes, we calculated the MS of the eigenmodes and the characteristic modes as shown in Figure 4. Furthermore, we calculated the relative differences in percentage between the MS of the eigenmodes and the characteristic modes as shown in Figure 5. The relative differences in percentage are calculated using

$$
\left|\frac{\lambda_{n}^{\text {cha }}-\lambda_{n}^{\text {eig }}}{\lambda_{n}^{\text {cha }}}\right| \cdot 100 \%
$$

In Figure 4, solid lines represent the MS of eigenmodes (EM) and scatters represent the MS of characteristic modes (CM). We select the first three eigenmodes and characteristic modes which have bigger MS. It can be observed from Figures 4 and 5 that the mesh densities hardly influence the numerical results. In other words, our numerical results are enough to ensure reasonable accuracy. More importantly, as shown in Figures 4 and 5, the MS of eigenmodes is similar to the MS of characteristic modes with slight differences. The slight differences are caused by the different currents of eigenmodes and characteristic modes. The current distributions of eigenmodes and characteristic modes at $141 \mathrm{MHz}$ (the first resonant frequency) are displayed in Figures 6 and 7. Figures 6 and 7 display the relative intensity of currents by the shade of the color as the colorbar shows, and all currents have been normalized to its maximum value to facilitate comparison. The black arrows in Figures 6 and 7 are used to indicate the direction of currents.
Comparing Figure 6 with Figure 7, it is observed that the eigenmodes and the characteristic modes share a one-to-one corresponding relationship just as the case of infinite circular cylinder and sphere, even the structures are noncanonical. However, unlike the case of infinite circular cylinder and sphere, the current distributions of eigenmodes and characteristic modes of PEC rectangular plate are not identical as observed from Figures 6 and 7. The following reasons cause the difference.

(1) The orthogonality of the eigenmodes and the characteristic modes is different as shown in (10) and (27).

(2) The current distributions of eigenmodes are generally complex, whereas the current distributions of characteristic modes are real, which are demonstrated in Section 2. That is to say that the current distributions of characteristic modes are equiphase on the surfaces of PEC bodies and the current distributions of eigenmodes are not equiphase. In order to further explain it, the phase distributions of currents of the first two eigenmodes at $141 \mathrm{MHz}$ are presented in Figure 8 . In Figure 8 , phase $\left(\vec{J}_{n x}^{\text {eig }}\right)$ represents the phase of the horizontal component of $\vec{J}_{n}^{\text {eig }}$, and phase $\left(\vec{J}_{n y}^{\text {eig }}\right)$ represents the phase of the vertical component of $\vec{J}_{n}^{\text {eig }}$. If $\vec{J}_{n}^{\text {eig }}$ is equiphase, phase $\left(\vec{J}_{n x}^{\text {eig }}\right)$ and phase $\left(\vec{J}_{n y}^{\text {eig }}\right)$ must be identical. As observed from Figure 8, the currents of eigenmodes are not equiphase.

Considering the MS, current distributions, and phase distributions of the current of eigenmodes and characteristic modes, it makes clear that (7) implies the approximative (not exact) relationship between the eigenvalues of eigenmodes and the characteristic values of characteristic modes. The currents of eigenmodes and characteristic modes are similar but not identical.

Besides, the radiation patterns of the eigenmodes and the characteristic modes are presented in Figures 9 and 10. The radiation patterns of the eigenmodes and the characteristic modes are similar, and this supports that 


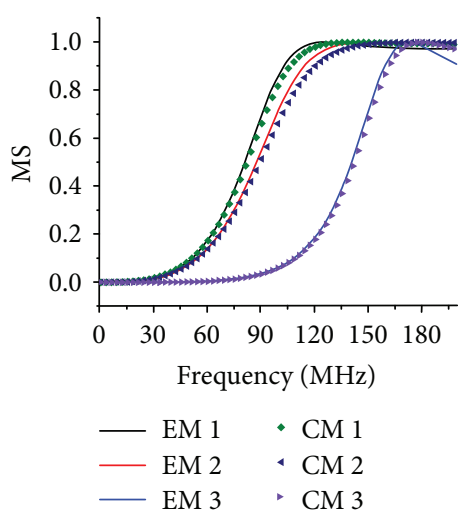

(a)

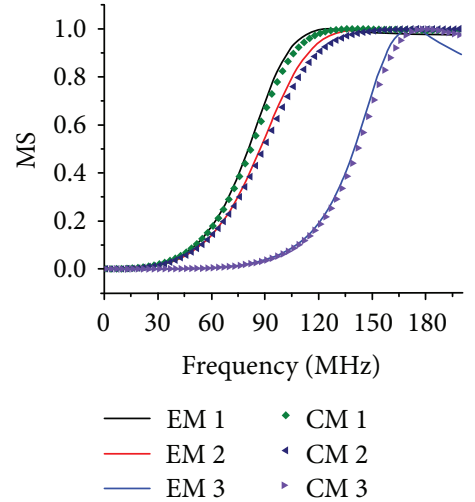

(b)

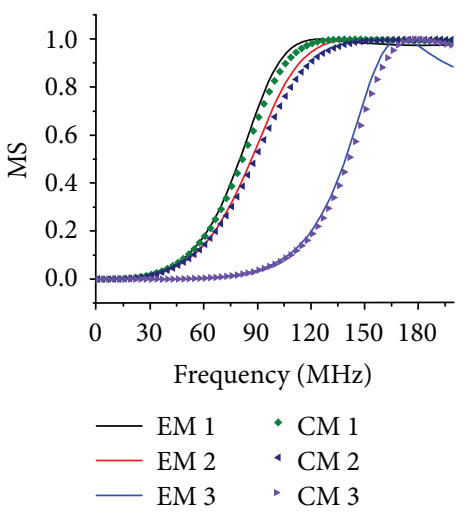

(c)

FIGURE 4: MS of the first three eigenmodes and characteristic modes. (a) Mesh 1. (b) Mesh 2. (c) Mesh 3.

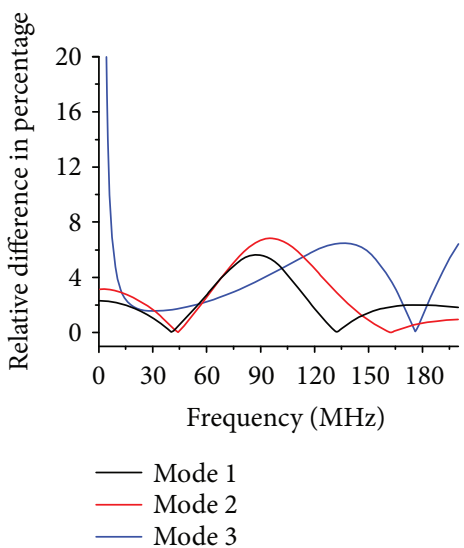

(a)

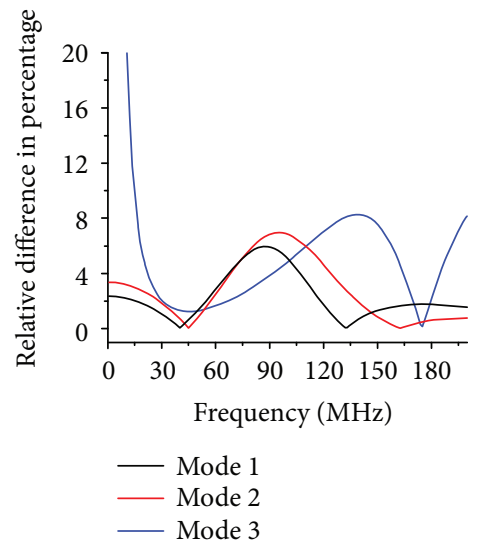

(b)

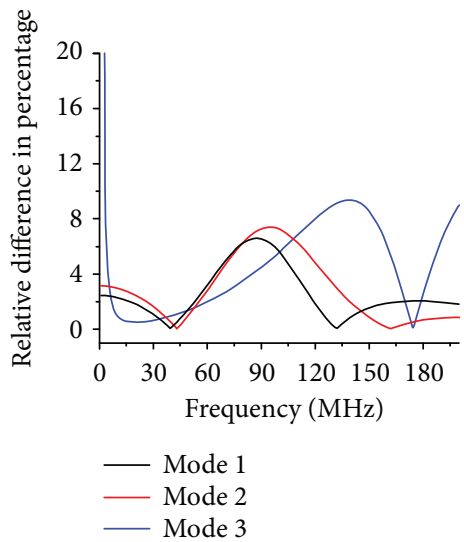

(c)

Figure 5: Relative difference of MS of the first three eigenmodes and characteristic modes. (a) Mesh 1. (b) Mesh 2. (c) Mesh 3.

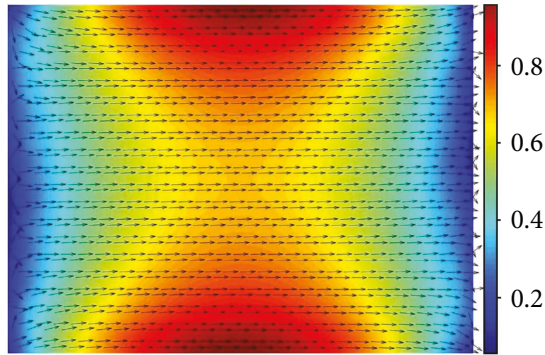

(a)

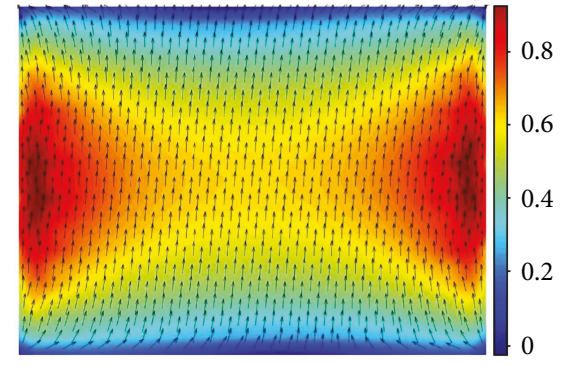

(b)

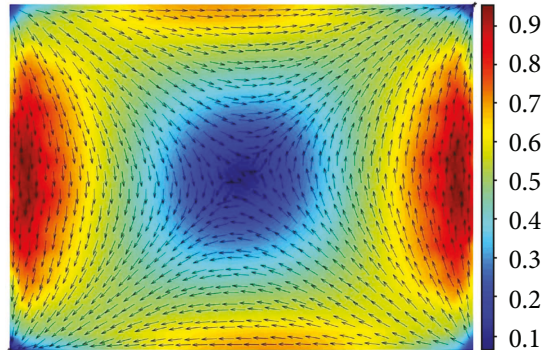

(c)

FIGURE 6: Current distributions of eigenmodes 1 to 3 at $141 \mathrm{MHz}$. (a) $\vec{J}_{1}^{\text {eig }}$. (b) $\vec{J}_{2}^{\text {eig }}$. (c) $\vec{J}_{3}^{\text {eig }}$.

the eigenmodes and the characteristic modes share a one-toone corresponding relationship.

The first three natural modes of the rectangular plate are presented in [22]. It is pointed out that the external resonant frequencies of the first three natural modes are $\omega_{c 1}=93.5+j 32.5 \mathrm{MHz}, \omega_{c 2}=99.7+j 39.4 \mathrm{MHz}$, and $\omega_{c 3}$ $=156.8+j 26.9 \mathrm{MHz}$. As shown in Figure 4, the first three external resonant frequencies of characteristic modes are
$141 \mathrm{MHz}, 179 \mathrm{MHz}$, and $184 \mathrm{MHz}$. Therefore, the external resonant frequencies of natural modes are different from those of characteristic modes. The reason why the external resonant frequencies of characteristic modes do not coincide with those of natural modes refers to $[8,15]$. The external resonances of natural modes generate the maximum radiated fields, while the external resonances of characteristic modes are not responsible for the maximally generated radiated 


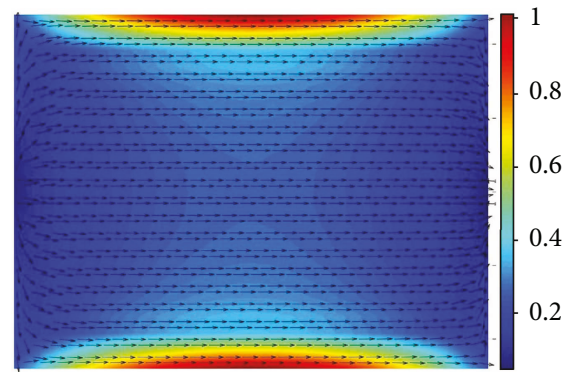

(a)

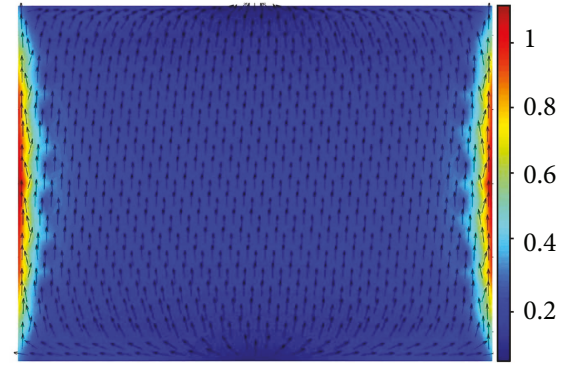

(b)

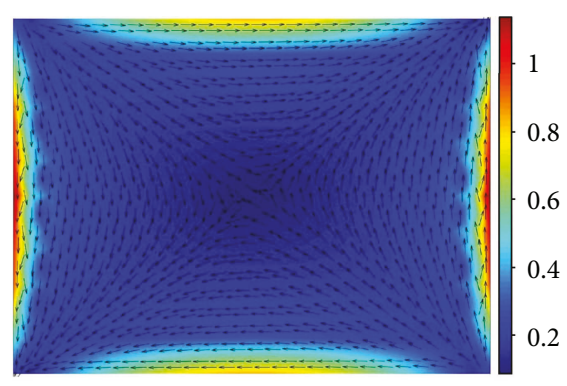

(c)

Figure 7: Current distributions of characteristic modes 1 to 3 at $141 \mathrm{MHz}$. (a) $\vec{J}_{1}^{\text {cha }}$. (b) $\vec{J}_{2}^{\text {cha }}$. (c) $\vec{J}_{3}^{\text {cha }}$.

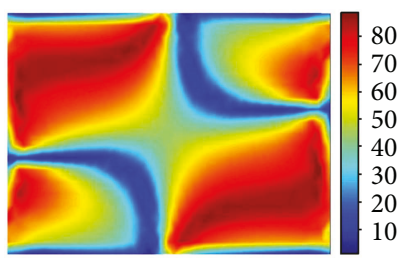

(a)

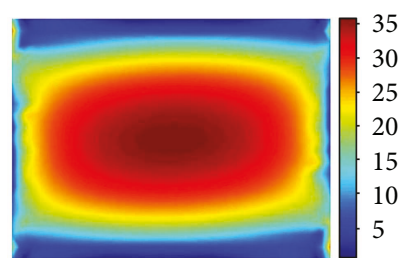

(b)

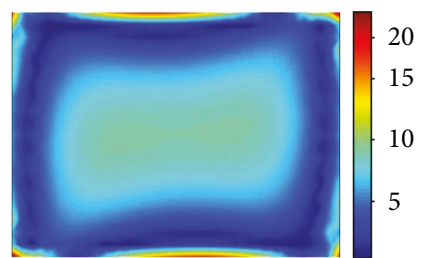

(c)

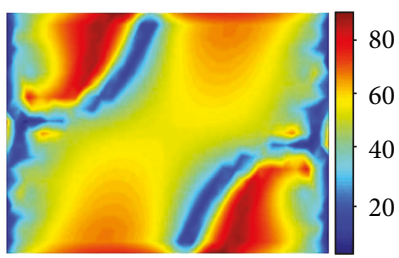

(d)

Figure 8: Phase distributions of two eigenmodes at $141 \mathrm{MHz}$. (a) Phase $\left(\vec{J}_{1 x}^{\mathrm{eig}}\right)$. (b) Phase $\left(\vec{J}_{1 y}^{\mathrm{eig}}\right)$. (c) Phase $\left(\vec{J}_{2 x}^{\mathrm{eig}}\right)$. (d) Phase $\left(\vec{J}_{2 y}^{\mathrm{eig}}\right)$.

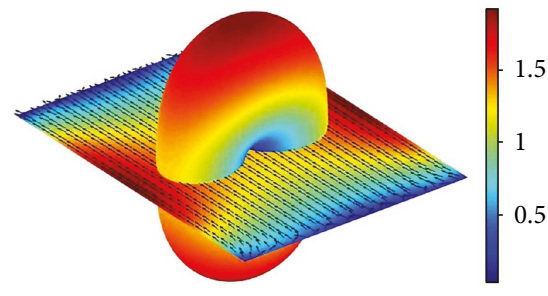

(a)

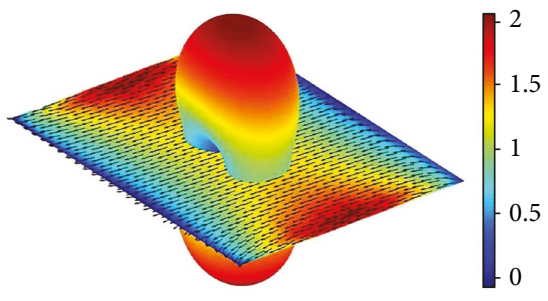

(b)

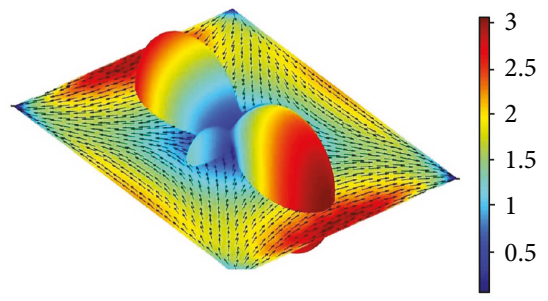

(c)

Figure 9: Radiation patterns of eigenmodes. (a) EM 1. (b) EM 2. (c) EM 3.

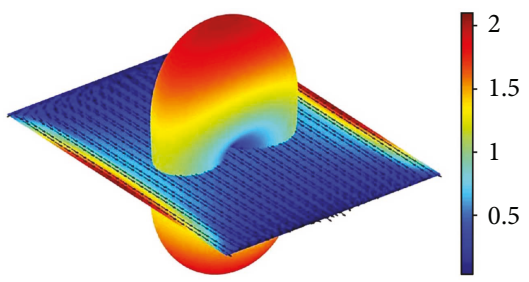

(a)

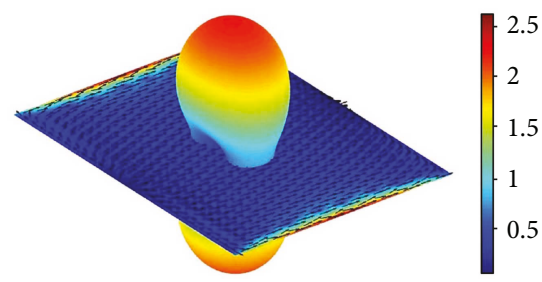

(b)

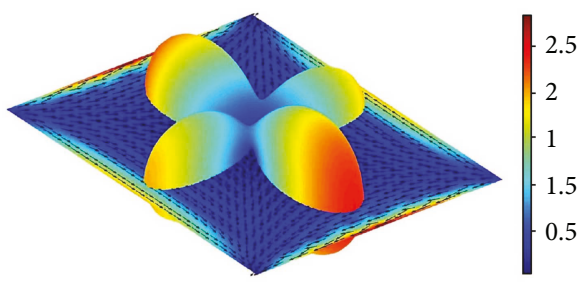

(c)

FIGURE 10: Radiation patterns of characteristic modes. (a) CM 1. (b) CM 2. (c) CM 3.

fields along certain directions [15]. A center-fed dipole antenna is discussed in [15] to prove that a resonant current does not imply that it is going to radiate more field intensity along a particular direction.

In fact, the similar analysis can be applied to penetrable bodies. On this occasion, the operator $\mathbf{Z}$ in (1) should be replaced with the operator of the integral equations for penetrable bodies. However, there are significant differences between the results of PEC bodies and penetrable bodies.
For example, concerning PEC bodies, it is found that the external resonant frequencies of natural modes are quite different from the external resonant frequencies of characteristic modes. But concerning penetrable bodies, the external resonant frequencies of natural modes coincide with the external resonant frequencies of characteristic modes $[18,26]$. Because of the limit of paper's length, we tend to discuss the relation between modes of penetrable bodies in future work. 


\section{Conclusion}

This paper specifies the corresponding relationships between eigenmodes, natural modes, and characteristic modes of PEC bodies. The characteristics of the electromagnetic power of natural modes for arbitrary PEC bodies are presented for the first time. It is found that the difference between the electric and magnetic energy of the natural mode is zero. By comparing the three kinds of modes of three typical structures, we found that there is a one-to-one corresponding relationship between the eigenmodes and the characteristic modes, as well as a one-to-many corresponding relationship between the eigenmodes and the natural modes. For several canonical structures (infinite circular cylinder and sphere), the current distributions of eigenmodes, natural modes, and characteristic modes are identical. For noncanonical structures, the current distributions of eigenmodes and characteristic modes are similar but not identical. Whether the structures are canonical or not, the internal resonant frequencies of natural modes coincide with those of characteristic modes, whereas the external resonant frequencies do not coincide.

\section{Appendix}

\section{Derivations of Eigenmodes of PEC Sphere}

Let us consider for instance the $\mathrm{TM}_{r}$ modes. The fields can be derived with $A_{r}$ given by [19].

$$
A_{r}=\left\{\begin{array}{c}
a_{n} \widehat{J}_{n}(k r) \\
b_{n} \widehat{H}_{n}^{(2)}(k r)
\end{array}\right\} P_{n}^{m}(\cos \theta)\left\{\begin{array}{c}
\cos m \varphi \\
\sin m \varphi
\end{array}\right\}, \quad r<a, r>a .
$$

The nonzero transverse electric and magnetic field components are

$$
\begin{aligned}
E_{\theta} & =\frac{1}{j \omega \mu \varepsilon} \cdot \frac{1}{r} \cdot \frac{\partial^{2} A_{r}}{\partial r \partial \theta} \\
& =\frac{1}{j \omega \mu \varepsilon r}\left\{\frac{a_{n}\left(\partial \widehat{J}_{n}(k r) / \partial r\right)}{b_{n}\left(\partial \widehat{H}_{n}^{(2)}(k r) / \partial r\right)}\right\} \frac{\partial P_{n}^{m}(\cos \theta)}{\partial \theta}\left\{\begin{array}{c}
\cos m \varphi \\
\sin m \varphi
\end{array}\right\}, \quad r<a, r>a, \\
E \varphi & =\frac{1}{j \omega \mu \varepsilon} \cdot \frac{1}{\sin \theta} \cdot \frac{\partial^{2} A_{r}}{\partial r \partial \varphi} \quad(\mathrm{A} .2) \\
& =\frac{1}{j \omega \mu \varepsilon r}\left\{\frac{a_{n}\left(\partial \widehat{J}_{n}(k r) / \partial r\right)}{b_{n}\left(\partial \widehat{H}_{n}^{(2)}(k r) / \partial r\right)}\right\} \frac{m P_{n}^{m}(\cos \theta)}{\sin \theta}\left\{\begin{array}{c}
-\sin m \varphi \\
\cos m \varphi
\end{array}\right\}, \\
r & <a, r>a, \\
H_{\theta} & =\frac{1}{\mu r} \cdot \frac{1}{\sin \theta} \cdot \frac{\partial A_{r}}{\partial \varphi} \quad(\mathrm{A} .3) \\
& =\frac{1}{\mu r}\left\{\begin{array}{l}
a_{n} \widehat{J}_{n}(k r) \\
b_{n} \widehat{H}_{n}^{(2)}(k r)
\end{array}\right\} \frac{m P_{n}^{m}(\cos \theta)}{\sin \theta}\left\{\begin{array}{l}
-\sin m \varphi \\
\cos m \varphi
\end{array}\right\}, \quad r<a, r>a,
\end{aligned}
$$

$$
\begin{aligned}
H_{\varphi} & =-\frac{1}{\mu r} \cdot \frac{\partial A_{r}}{\partial \theta} \\
& =\frac{1}{\mu r}\left\{\begin{array}{c}
a_{n} \widehat{J}_{n}(k r) \\
b_{n} \widehat{H}_{n}^{(2)}(k r)
\end{array}\right\} \frac{\partial P_{n}^{m}(\cos \theta)}{\partial \theta}\left\{\begin{array}{c}
\cos m \varphi \\
\sin m \varphi
\end{array}\right\}, \quad r<a, r>a .
\end{aligned}
$$

Applying the boundary conditions on the surface of sphere,

$$
\begin{aligned}
& \widehat{r} \times\left[\left(\widehat{\theta} H_{\theta}+\widehat{\varphi} H_{\varphi}\right)\left|S^{+}-\left(\widehat{\theta} H_{\theta}+\widehat{\varphi} H_{\varphi}\right)\right|_{S^{-}}\right]=\vec{J}_{m n}^{\text {eig,TM }}, \\
& \hat{r} \times\left[\left(\widehat{\theta} E_{\theta}+\widehat{\varphi} E_{\varphi}\right)\left|S^{+}-\left(\widehat{\theta} E_{\theta}+\widehat{\varphi} E_{\varphi}\right)\right|_{S^{-}}\right]=0,
\end{aligned}
$$

where $S^{+}$and $S^{-}$represent the external and internal surface of sphere, respectively.

Substituting (A.2), (A.3), (A.4), and (A.5) into (A.6) and (A.7) gives

$$
\begin{aligned}
& \vec{J}_{m n}^{\text {eig,TM }}=C_{\mathrm{EM}}^{\mathrm{TM}}\left[\widehat{\theta} \frac{\partial P_{n}^{m}(\cos \theta)}{\partial \theta}\left\{\begin{array}{l}
\cos m \phi \\
\sin m \phi
\end{array}\right\}\right. \\
& \left.+\widehat{\phi} \frac{m P_{n}^{m}(\cos \theta)}{\sin \theta}\left\{\begin{array}{c}
-\sin m \phi \\
\cos m \phi
\end{array}\right\}\right], \\
& {\left[b_{n} \frac{\partial \widehat{H}_{n}^{(2)}(k r)}{\partial r}-a_{n} \frac{\partial \widehat{J}_{n}(k r)}{\partial r}\right]_{r=a}=0}
\end{aligned}
$$

where

$$
C_{\mathrm{EM}}^{\mathrm{TM}}=\frac{b_{n} \widehat{H}_{n}^{(2)}(k a)-a_{n} \widehat{J}_{n}(k a)}{\mu a} .
$$

Finally, substituting (A.2), (A.3), and into (4), it is found that

$$
v_{m n}^{\mathrm{TM}}=-\frac{\left[\left(\partial \widehat{J}_{n}(k r) / \partial r\right)\left(\partial \widehat{H}_{n}^{(2)}(k r) / \partial r\right)\right]_{r=a}}{j \omega \varepsilon\left[\left.\left(\partial \widehat{J}_{n}(k r) / \partial r\right)\right|_{r=a} \widehat{H}_{n}^{(2)}(k a)-\left.\widehat{J}_{n}(k a)\left(\partial \widehat{H}_{n}^{(2)}(k r) / \partial r\right)\right|_{r=a}\right]} .
$$

Following the similar procedure, it can be obtained that the eigenvalues and currents of $\mathrm{TE}_{r}$ modes are

$$
\begin{gathered}
\vec{J}_{m n}^{\mathrm{eig}, \mathrm{TE}}=C_{\mathrm{EM}}^{\mathrm{TE}}\left[-\widehat{\theta} \frac{m P_{n}^{m}(\cos \theta)}{\sin \theta}\left\{\begin{array}{c}
-\sin m \phi \\
\cos m \phi
\end{array}\right\}\right. \\
\left.+\widehat{\phi} \frac{\partial P_{n}^{m}(\cos \theta)}{\partial \theta}\left\{\begin{array}{c}
\cos m \phi \\
\sin m \phi
\end{array}\right\}\right], \\
\widehat{v}_{m n}^{\mathrm{TE}}=j \omega \mu \frac{\widehat{J}_{n}(k a) \widehat{H}_{n}^{(2)}(k a)}{\left[\widehat{J}_{n}(k a)\left(\partial \widehat{H}_{n}^{(2)}(k r) / \partial r\right)=\widehat{H}_{n}^{(2)}(k a)\left(\partial \widehat{J}_{n}(k r) / \partial r\right)\right]_{r=a}},
\end{gathered}
$$


where

$$
C_{\mathrm{EM}}^{\mathrm{TE}}=\frac{\left[b_{n}\left(\partial \widehat{H}_{n}^{(2)}(k r) / \partial r\right)-a_{n}\left(\partial \widehat{J}_{n}(k r) / \partial r\right)\right]_{r=a}}{j \omega \mu \varepsilon a} .
$$

\section{Conflicts of Interest}

The authors declare that they have no conflicts of interest.

\section{References}

[1] R. F. Harrington, Time-Harmonic Electromagnetic Fields, McGraw-Hill, 1961.

[2] R. F. Harrington, Field Computation by Moment Methods, Macmillan, 1968.

[3] A. Ramm, "Theoretical and practical aspects of singularity and eigenmode expansion methods," IEEE Transactions on Antennas and Propagation, vol. 28, no. 6, pp. 897-901, 1980.

[4] C. E. Baum, "The singularity expansion method," Transient Electromagnetic Fields, vol. 10, pp. 129-179, 1976.

[5] M. Vogel, G. Gampala, D. Ludick, and C. J. Reddy, "Characteristic mode analysis: putting physics back into simulation," IEEE Antennas and Propagation Magazine, vol. 57, no. 2, pp. 307-317, 2015.

[6] R. F. Harrington and J. R. Mautz, "Theory and computation of characteristic modes for conducting bodies," DTIC Document, Tech. Rep., Electrical Engineering Department, Syracuse University, Syracuse, NY, USA, 1970.

[7] J. V. Bladel, Electromagnetic Fields, Hemisphere Pub. Corp, 1979.

[8] T. Bernabeu-Jimenez, A. Valero-Nogueira, F. Vico-Bondia, and A. A. Kishk, "A comparison between natural resonances and characteristic mode resonances of an infinite circular cylinder," IEEE Transactions on Antennas and Propagation, vol. 65, no. 5, pp. 2759-2763, 2017.

[9] R. J. Garbacz, A Generalized Expansion for Radiated and Scattered Fields, [Ph.D. thesis], The Ohio State University, Columbus, OH, USA, 1968.

[10] R. B. Adler, L. J. Chu, and R. M. Fano, Electromagnetic Energy Transmission and Radiation, Wiley, 1960.

[11] H. Alroughani, An Appraisal of the Characteristic Modes of Composite Objects, 2013.

[12] R. Rezaiesarlak and M. Manteghi, Chipless RFID: Design Procedure and Detection Techniques, Springer, Cham, 2014.

[13] M. Cabedo-Fabrs, E. Antonino-Daviu, T. Bernabeu-Jimnez, and M. Ferrando-Bataller, "Review and application of the theory of characteristic modes for open radiating structures," in 2015 European Microwave Conference (EuMC), pp. 5-8, Paris, France, 2015.

[14] T. Bernabeu-Jimnez, A. Valero-Nogueira, F. Vico-Bondia, and A. A. Kishk, "Relation between characteristic modes and complex natural resonances," in 2015 IEEE International Symposium on Antennas and Propagation \& USNC/URSI National Radio Science Meeting, pp. 452-453, Vancouver, BC, Canada, 2015.

[15] T. K. Sarkar, E. L. Mokole, and M. Salazar-Palma, "An expose on internal resonance, external resonance, and characteristic modes," IEEE Transactions on Antennas and Propagation, vol. 64, no. 11, pp. 4695-4702, 2016.
[16] E. A. Daviu, Analysis and Design of Antennas for Wireless Communications Using Modal Methods, [Ph.D. thesis], Universitat Politècnica de València, Valencia, Spain, 2008.

[17] K. F. Warnick, Numerical Analysis for Electromagnetic Integral Equations, Arctech House, Norwood, MA, USA, 2008.

[18] R. Lian, J. Pan, and S. Huang, "Alternative surface integral equation formulations for characteristic modes of dielectric and magnetic bodies," IEEE Transactions on Antennas and Propagation, vol. 65, no. 9, pp. 4706-4716, 2017.

[19] J. Jin, Theory and Computation of Electromagnetic Fields, Wiley, 2010.

[20] E. Heyman and L. Felsen, "Creeping waves and resonances in transient scattering by smooth convex objects," IEEE Transactions on Antennas and Propagation, vol. 31, no. 3, pp. 426-437, 1983.

[21] E. Heyman and L. Felsen, "A wavefront interpretation of the singularity expansion method," IEEE Transactions on Antennas and Propagation, vol. 33, no. 7, pp. 706-718, 1985.

[22] J. K. Lawrence, On the Use of Natural-Mode Basis Functions for Electromagnetic Analysis of Arbitrary Conducting Surfaces, [M.S. thesis], Clemson University, Clemson, SC, USA, 2010.

[23] S. Rao, D. Wilton, and A. Glisson, "Electromagnetic scattering by surfaces of arbitrary shape," IEEE Transactions on Antennas and Propagation, vol. 30, no. 3, pp. 409-418, 1982.

[24] R. Harrington and J. Mautz, "Computation of characteristic modes for conducting bodies," IEEE Transactions on Antennas and Propagation, vol. 19, no. 5, pp. 629-639, 1971.

[25] P. Yla-Oijala and M. Taskinen, "Calculation of CFIE impedance matrix elements with RWG and n/spl times/RWG functions," IEEE Transactions on Antennas and Propagation, vol. 51, no. 8, pp. 1837-1846, 2003.

[26] F.-G. Hu and C.-F. Wang, "Integral equation formulations for characteristic modes of dielectric and magnetic bodies," IEEE Transactions on Antennas and Propagation, vol. 64, no. 11, pp. 4770-4776, 2016. 


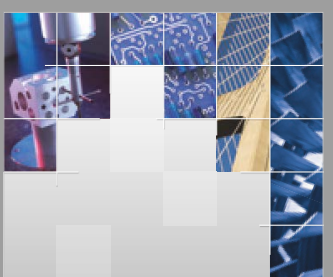

\section{Enfincering}
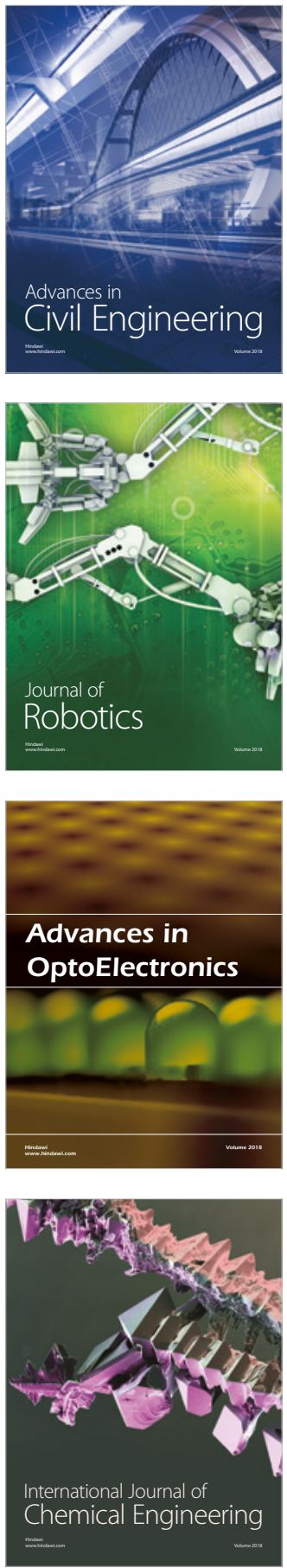

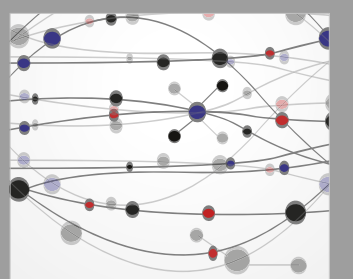

\section{Rotating \\ Machinery}

The Scientific World Journal

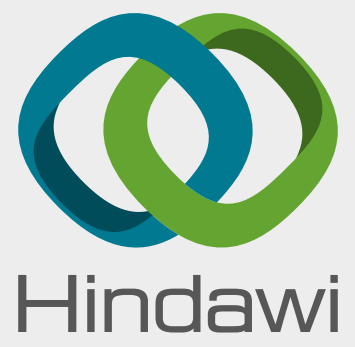

Submit your manuscripts at

www.hindawi.com
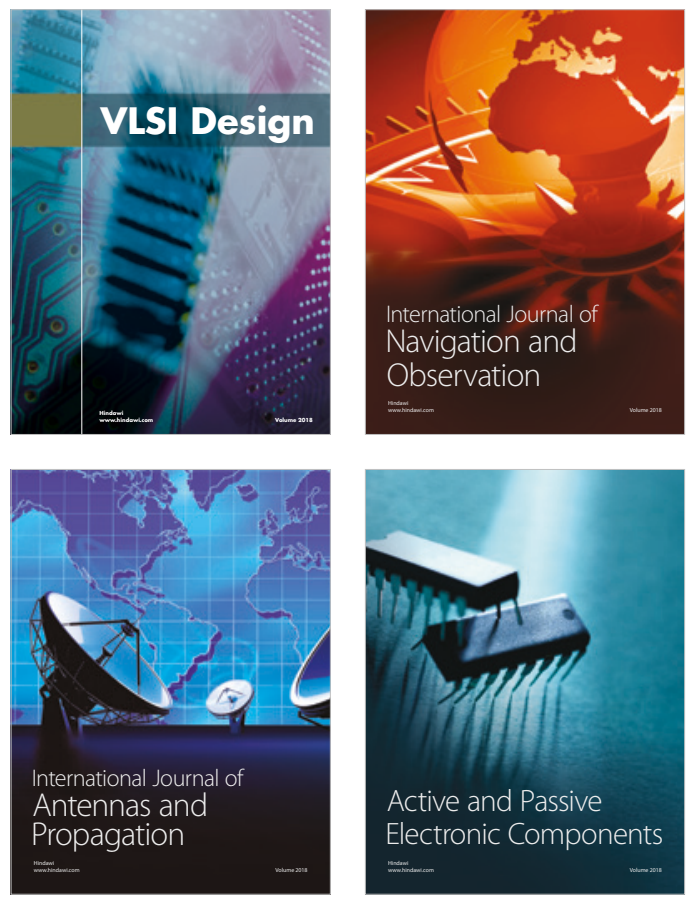
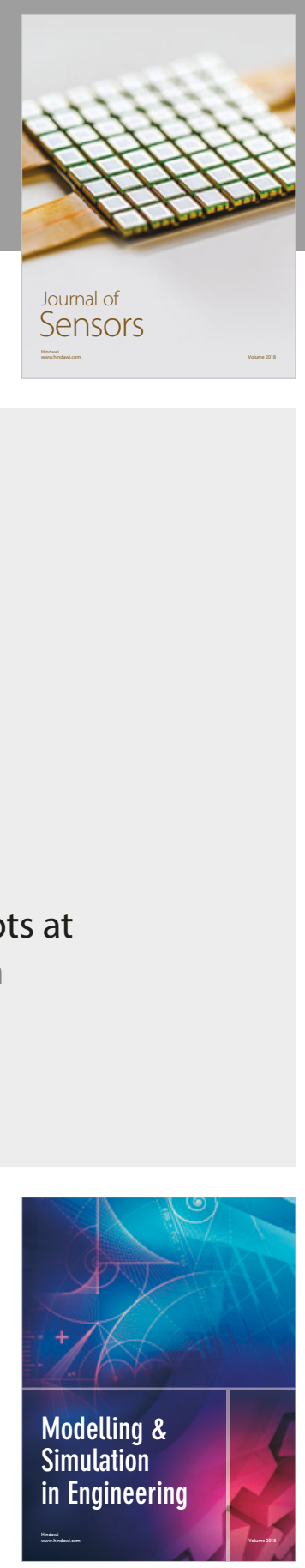

\section{Advances \\ Multimedia}
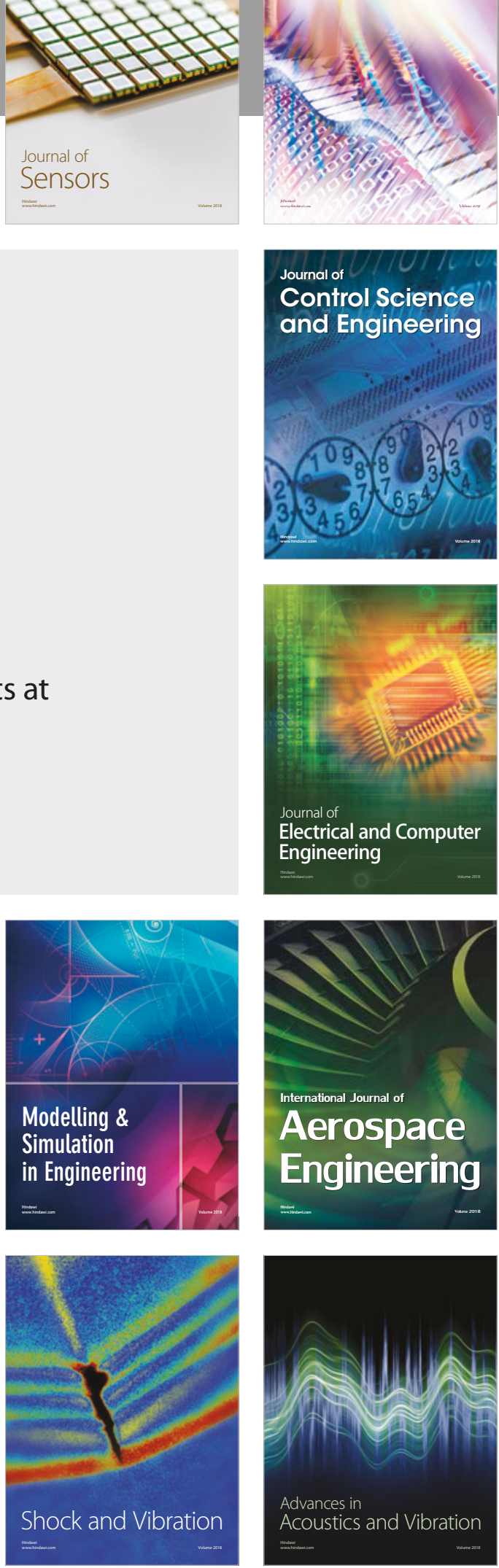